\title{
Research article \\ Clinical usefulness of eosinopenia in differentiating COVID-19 versus other flu-like illness in outpatient set-up
}

\author{
Koushik Muthu Raja M., Anand R., Vinod V. G., Rajagopalan B., Chandrasekar C., Dhanasekar T. \\ Department of Respiratory Medicine, Sri Ramachandra Medical College and Research Institute, Porur Chennai, Tamil Nadu, \\ India
}

(Received: February $2021 \quad$ Revised: June $2021 \quad$ Accepted: June 2021)

Corresponding author: Vinod V.G. Email: v.g.vinod14@gmail.com

\begin{abstract}
Introduction and Aim: Corona virus disease 2019 (Covid-19) is a highly infectious disease caused by severe acute respiratory syndrome coronavirus 2 (SARS-CoV-2). COVID-19 has affected over 200 countries and territories. The aim of the present study is to evaluate the role of eosinopenia in differentiating COVID-19 suspect from other flu-like illnesses in an outpatient setting.

Materials and Methods: It was a retrospective case control study in which data of 1252 suspected COVID 19 patients who visited the fever clinic were enrolled. Among this, 701 patients were found to have COVID-19 pneumonia and 551 had other flu-like illnesses. CBC (complete blood count) and CRP (C- reactive protein) were taken in all patients along with chest $\mathrm{x}$-ray as routine protocol.

Results: Among the 701 positive patients, eosinopenia was seen in 527 patients and had a sensitivity of $75.2 \%$ and specificity of $68.6 \%$. When combined with other lab parameters such as the increased CRP and lymphopenia, the specificity rises up to $78.6 \%$.

Conclusion: Our study shows that eosinopenia as an independent variable, is a reliable marker to differentiate COVID-19 suspect patients from other flu-like illnesses on outpatient basis, however when combined with other parameters like lymphopenia and CRP the specificity increases further.
\end{abstract}

Keywords: COVID; eosinopenia; lymphopenia; flu-like illnesses.

\section{INTRODUCTION}

$\longrightarrow$ orona virus disease 2019 (Covid-19) is a highly infectious disease caused by severe acute respiratory syndrome. Corona virus 2 (SARS-Cov-2) was first reported in Wuhan, Hubei Province, China (1). On March 11, 2020, the world health Organization (WHO) declared this ongoing outbreak as global pandemic (2). As of January 31, 2021, COVID-19 has affected over 200 countries and territories and in India the COVID-19 has risen up to 10,746,183 (Nearly 10.8 million cases) and leading to 154,649 deaths. According to WHO guidelines $(3,4)$, the suspected Covid-19 cases should have any one of the following features (i) recent travel history to the containment zone or close contact with laboratory confirmed case (ii) fever and / or respiratory symptoms (iii) radiographic evidence of pneumonia.

A key to slowdown the spread towards the outbreak containment is by identifying suspected patients from other patients with flu-like illness for isolation or further diagnostic examination towards timely treatment. The major difficulty faced at the routine outpatient set-up was how to accurately and effectively sort suspected COVID-19 patients from other pneumonia (or) respiratory infection who presented with acute upper or lower respiratory infection symptoms. The significant delay in diagnosis and subsequent quarantine process leading to cross infection and effective ways should be carried out at the triage to sort out suspected COVID -19 cases from other patients with similar symptoms (2). Here in this study, we plan to identify simple and quickly available laboratory parameters as biomarkers for differentiating COVID 19 patients from patients with other flu-like illness.

\section{MATERIALS AND METHODS}

\section{Patient selection}

This was a retrospective case-control study where the data of 2000 patients who visited the fever clinic of Sri Ramachandra Medical College and Hospital, Chennai from April 2020 to August 2020 prior to any antibiotic (or) antiviral treatment were collected and analyzed. All suspected COVID-19 patients visiting the fever clinic with any one of the following symptoms (Fever, dry cough, sore throat, myalgia, and breathing difficulty) and people with recent travel history to the containment zone or close contact with laboratory confirmed cases and radiographic evidence of pneumonia were considered for inclusion in this study. Those patients without routine blood examinations, patients refusing for routine blood tests or wanted to go other hospitals for further testing, and patients who already took throat swab or computed tomography (CT) chest from outside hospital were excluded. Finally, a total of 
1252 cases were included in our study based on the inclusion and exclusion criteria.

\section{Procedure}

All the 1252 included patients' data were collected from institutional records which included complete blood count, C-reactive protein (CRP) levels, chest xray and also RT-PCR (nucleic acid testing) for SARS-COV-2. Among the 1252 patients, 701 patients were tested positive and considered as covid19 cases and 551 were tested negative and are considered as controls. Routine blood analysis and CRP were performed in clinical laboratory of Sri Ramachandra Hospital, Chennai. Blood biomarkers tests had been carried out by Beckman Coulter DXH -800 hematology analyzer. The laboratory confirmation of SARS-COV-2 infection had been carried out by RT-PCR through amplifying ORF I (Open Reading frames) gene and N (Nucleocapsid) gene of SARS-COV-2 from nasopharyngeal specimens of all patients.

\section{Interpretation of results}

The patient is considered to have eosinopenia if eosinophil count is less than 1.0 (normal 1-6) and elevated CRP is when the serum CRP levels are elevated more than $0.8 \mathrm{mg} / \mathrm{dl}$. Lymphopenia is defined when the lymphocytes count is less than 20 $\%$ (normal $20-40 \%$ ).

\section{RESULTS}

The data were entered and analyzed with SPSS Version 16. The median age for COVID positive patients is 45.6 years [41-60 years]. The table 1 shows the basic demographic data and table 2 shows the laboratory parameters between the two groups. Among the three biomarkers (CRP, Lymphocyte and eosinophils) that have been compared in the study, 527 COVID-19 patients had eosinopenia. Eosinopenia as a single variable has a sensitivity and specificity which is $75.2 \%$ and $68.6 \%$ (Table 3 ). The lymphopenia has a sensitivity and specificity of $47.2 \%$ and $38.8 \%$ while the increased CRP (>0.8) has a sensitivity and specificity of $61.2 \%$ and $11.8 \%$.

The predictive capacity of those significant biomarkers (eosinopenia, elevated CRP and lymphopenia) both in the univariate and multivariate logistic analyses were done. Since, less than $10 \%$ of the participants had decreased neutrophils, this parameter was not included in the diagnostic performance analysis. Among the three remaining biomarkers, eosinopenia produced higher sensitivity of $75.2 \%$, specificity of $68.6 \%$ and PPV (Positive Predictive Value) of $75.3 \%$. Then the eosinopenia was further singly or doubly or triply combined with the other 2 biomarkers (CRP and lymphocyte). We found that the combination of eosinopenia and increased CRP yielded the highest specificity of $74.6 \%$ with PPV of $70.2 \%$. We also observed that when we combine three variables eosinopenia, lymphopenia and increased CRP the specificity of these biomarkers reaches to $78 \%$ with PPV of $62.1 \%$ (Table 3 and Table 4).

Table 1: Basic demographic data of the included participants $(\mathrm{N})$

\begin{tabular}{|c|c|c|c|}
\hline \multicolumn{2}{|c|}{ Variable } & Number (N) & Percentage (\%) \\
\hline \multirow{3}{*}{$\begin{array}{c}\text { Age in } \\
\text { years }\end{array}$} & $<20$ & 23 & 3.2 \\
\cline { 2 - 4 } & $21-40$ & 244 & 34.8 \\
\cline { 2 - 4 } & $41-60$ & 297 & 41.5 \\
\cline { 2 - 4 } & $>60$ & 137 & 19.5 \\
\hline \multirow{3}{*}{ Gender } & Male & 405 & 57.7 \\
\cline { 2 - 4 } & Female & 296 & 42.2 \\
\hline
\end{tabular}

Table 2: Comparison of biomarkers between patients with and without covid-19

\begin{tabular}{|c|c|c|c|c|c|}
\hline S. No. & \multicolumn{2}{|c|}{ Variable } & COVID-19 Positive & COVID-19 Negative & Total \\
\hline \multirow{3}{*}{1} & \multirow{2}{*}{ Patients } & Total & 701 & 551 & 1252 \\
\cline { 3 - 6 } & & Male & 405 & 253 & 658 \\
\cline { 2 - 5 } & & Female & 296 & 298 & 594 \\
\hline 2 & \multicolumn{2}{|c|}{ Total Count $(<4000 />11000)$} & 143 & 196 & 339 \\
\hline 3 & \multicolumn{2}{|c|}{ Eosinopenia $(<1)$} & 527 & 173 & 700 \\
\hline 4 & \multicolumn{2}{|c|}{ Lymphopenia (<20) } & 331 & 337 & 668 \\
\hline 5 & \multicolumn{2}{|c|}{ CRP $(>0.8)$} & 429 & 486 & 915 \\
\hline
\end{tabular}


Table 3: Diagnostic performance of individual or combined serum biomarkers in differentiating COVID -19 patients (701) from control patients (551)

\begin{tabular}{|c|c|c|c|c|c|c|}
\hline Variables & Sensitivity & Specificity & $\begin{array}{c}\text { Positive } \\
\text { Predictive value }\end{array}$ & $\begin{array}{c}\text { Negative } \\
\text { Predictive value }\end{array}$ & $\begin{array}{c}\text { \% False } \\
\text { Positive }\end{array}$ & $\begin{array}{c}\text { \% False } \\
\text { Negative }\end{array}$ \\
\hline Eosinopenia (EOS) $(<1)$ & 75.2 & 68.6 & 75.3 & 68.5 & 31.4 & 24.8 \\
\hline $\begin{array}{c}\text { Total Leucocyte count } \\
(\text { TLC) }(<4000 />11000)\end{array}$ & 20.4 & 64.4 & 42.2 & 38.9 & 35.6 & 79.6 \\
\hline $\begin{array}{c}\text { Lymphocytes (LYMP) } \\
(<20)\end{array}$ & 47.2 & 38.8 & 49.6 & 36.6 & 61.2 & 52.8 \\
\hline CRP (>0.8) & 61.2 & 11.8 & 46.9 & 19.3 & 88.2 & 38.8 \\
\hline Neutrophils (<40) & 59.9 & 15.2 & 47.4 & 23.0 & 84.7 & 40.1 \\
\hline EOS (<1)+ CRP (>0.8) & 54.4 & 74.6 & 70.2 & 54.9 & 39.4 & 45.6 \\
\hline $\begin{array}{c}\text { EOS (<1)+ TLC } \\
(<4000 />11000)\end{array}$ & 15.5 & 84.9 & 56.8 & 44.2 & 15.1 & 84.5 \\
\hline EOS $(<1)+$ LYMP $(<20 \%)$ & 36.9 & 77.0 & 67.1 & 49.0 & 23 & 63.1 \\
\hline $\begin{array}{c}\text { EOS (<1)+ Neutrophils } \\
(<40)\end{array}$ & 50.4 & 63.0 & 70.3 & 53.60 & 27.0 & 49.6 \\
\hline $\begin{array}{c}\text { EOS }(<1)+\text { CRP }(>0.8)+ \\
\text { LYMP }(<20)\end{array}$ & 28.2 & 78.0 & 62.1 & 46.1 & 22.0 & 71.8 \\
\hline
\end{tabular}

Table 4: Comparison of diagnostic performance of eosinopenia and other variables

\begin{tabular}{|c|c|c|c|c|c|c|}
\hline Results & Sensitivity & Specificity & $\begin{array}{c}\text { Positive } \\
\text { Predictive value }\end{array}$ & $\begin{array}{c}\text { Negative } \\
\text { Predictive Value }\end{array}$ & $\begin{array}{c}\text { \% False } \\
\text { Positive }\end{array}$ & $\begin{array}{c}\text { \% False } \\
\text { Negativity }\end{array}$ \\
\hline Eosinopenia (<1) & 75.2 & 68.6 & 75.3 & 68.5 & 31.4 & 24.8 \\
\hline CRP $(>0.8)$ & 61.2 & 11.8 & 46.9 & 19.3 & 88.2 & 38.8 \\
\hline $\begin{array}{c}\text { Eosinopenia (<1)+CRP } \\
(>0.8)\end{array}$ & 54.4 & 73.6 & 70.2 & 54.9 & 39.4 & 45.6 \\
\hline $\begin{array}{c}\text { Eosinopenia (<1)+CRP } \\
(>0.8)+\text { +Lymphopenia } \\
(<20 \%)\end{array}$ & 28.2 & 78 & 62.1 & 46.1 & 22 & 71.8 \\
\hline
\end{tabular}

\section{DISCUSSION}

Eosinophils are circulating and tissue-resident leukocytes which show pro-inflammatory, immunoregulatory and antiviral effects (5). Eosinopenia has been reported in different medical conditions such as bacterial infections, acute inflammation, and stress or corticosteroid therapy. In COVID-19, eosinopenia may be related to the migration of these cells into the peripheral tissues or to a decreased production of eosinophils into the bone marrow, due to the inflammatory state (6). Chusid in his study stated that though eosinophil was thought initially as a key effector cell of allergy, it has antiviral properties which reduce inflammation (7) which is the same finding with the results of our study. Lymphopenia could be another potential explanation for eosinopenia. A large number of patients with COVID-19 have a decreased lymphocytes count during their presentation, and Th2 (T-helper 2)subtype lymphocytes are responsible for the production of IL-5 (interleukin 5), which stimulates the activation and the production of eosinophils. The WHO interim Guidelines $(3,4)$ is followed worldwide which defines the suspected patients based on the epidemiological history and clinical symptoms.

In this COVID-19 pandemic situation, it is difficult to differentiate suspected COVID-19 patients from other patients with similar flu-like illness in routine outpatient (OP) set-up. In light of this rationale, we performed this analysis. Our case-control study showed that eosinopenia as an independent variable is a reliable marker to differentiate COVID-19 suspects from other flu-like illnesses on OP basis and the combination of eosinopenia with other markers like lymphopenia and CRP has increased the specificity up to $78 \%$. Vasiliki et al., evaluated the role of eosinophils in their study and reported that lower eosinophil count could be associated with worst outcomes and prolonged hospitalization of moderate to severe COVID-19 patients (8). The findings of the study by Haider concluded that eosinopenia is an intelligible test favoring the diagnosis of COVID-19 patients (9). The same finding was reported in our study stating that eosinopenia was found in suspected COVID-19 patients during their first medical visit suggesting that it might be an early event of COVID-19 infection prior to emergence of clinical and radiological findings. So, eosinopenia as an independent parameter in routine blood test can be used to differentiate suspected COVID-19 patients from other patients with similar symptoms. The identified suspected patients could be subjected to HRCT (High resolution computed tomography) thorax and laboratory definitive diagnosis (nasopharyngeal swab for SARS-CoV-2 RT-PCR) followed by isolation in separate wards to prevent potential spread of COVID-19 and to prevent cross 
infection among health care workers. Our findings of eosinopenia, increased CRP and lymphopenia in all suspected patients can be used as a supplement in the routine outpatient set-up as these tests are readily available and are cost-effective and time-consuming. Eosinopenia can be considered as a reliable early diagnostic marker for COVID-19 infection (10) which is in line with our study. A cut-off level of $10 / \mu \mathrm{L}$ eosinopenia can be considered as a useful test in suspected COVID-19 patients with negative PCR and suspicious chest CT (11) which is also evident in our study.

Our study has several limitations: 1 . It is a retrospective case control study 2. Presence of selection biases due to hospital-based case control study 3 . The nucleic acid test could yield false negative results mainly due to insufficient viral load below detection limit in the nasopharyngeal swabs and there is also a possibility of viral disintegration during sample collection, transport and processing 4 . The outcomes of the COVID-19 negative cases (controls) were not followed up in this study.

\section{CONCLUSION}

In summary, our study shows that eosinopenia as an independent variable, has good sensitivity and specificity, which helps in early diagnosis and prevention of cross infection among healthcare workers during triaging process. Hence, eosinopenia as an independent variable can be used as a reliable marker to differentiate COVID-19 suspect patients from other flu-like illnesses in an outpatient set-up.

\section{CONFLICT OF INTEREST}

Authors declare no conflict of interest.

\section{REFERENCES}

1. Xia, Z. Eosinopenia as an early diagnostic marker of COVID-19 at the time of the epidemic. E Clinical Medicine. 2020; 23:100398.

2. Lia, Q., Dinga, X., Xiab, G., Chend, H. G., Chena, F., Geng, Z. Eosinopenia and elevated C-reactive protein facilitate triage of COVID-19 patients in fever clinic: A retrospective case-control study. E Clinical Medicine. 2020; 23: 100375.

3. World Health Organization. Rolling updates on corona virus disease (COVID-19) (Updated 18 April 2020). https://www.who.int/emergencies/diseases/novel-coronavirus-2019/events-as-they-happen.AccessedApr21,2020

4. World Health Organization. Corona virus disease 2019 (COVID-19) situation report_74. https://www.who.int/docs/defaultsource/coronaviruse/situation-reports/20200403-sitrep-74covid-19-mp.pdf?sfvrsn=4e043d03_10.AccessedApr4,2020

5. Djangang, N. N., Peluso, L., Talamonti, M., Izzi, A., Gevenois, P. A., Garuf, A., et al., Eosinopenia in COVID-19 patients: A retrospective analysis. Microorganisms. 2020; 8: 1929.

6. Tanni, F., Akker, E., Zaman, M. M., Figueroa, N., Tharian, B., Hupart, K. H. Eosinopenia and COVID-19. The Journal of the American Osteopathic Association. 2020. 120: 504508.

7. Chusid, M. J. Eosinophils: friends or foes? The journal of allergy and clinical immunology. Practice. 2018; 6: 14391444.
8. Vasiliki, E. G., Nikolaos, G., Christos, D., Serena, V., Dimitrios, D., Evangelos, D., et al., The impact of peripheral eosinophil counts and eosinophil to lymphocyte ratio (ELR) in the clinical course of COVID-19 patients: A retrospective study. In Vivo. 2021; 35(1): 641-648.

9. Haider, S. D. Eosinophil count as the predictor of main outcomes of COVID 19: case-control study. European Journal of Molecular \& Clinical Medicine. 2020; 7(2): 4429.

10. Soni, M. Evaluation of eosinopenia as a diagnostic and prognostic indicator in COVID-19 infection. International Journal of Laboratory Hematology. 2020; 00: 1-5.

11. Outh, R., Boutin, C., Gueudet, P., Suzuki, M., Saada, M., Aumatre, H., et al., Eosinopenia $<100 / \mathrm{mL}$ as a marker of active COVID-19: An observational prospective study. Journal of Microbiology, Immunology and Infection. 2021; $54 ; 61-68$. 